\title{
International Classification of Diseases
}

National Cancer Institute

\section{Source}

National Cancer Institute. International Classification of Diseases. NCI Thesaurus. Code C49474.

A system of categories to which morbid entries are assigned according to established criteria. Included is the entire range of conditions in a manageable number of categories, grouped to facilitate mortality reporting. It is produced by the World Health Org anization. The Clinical Modifications, produced by the United States Dept. of Health and Human Services, are larger extensions used for morbidity and general epidemiological purposes, primarily in the U.S. 